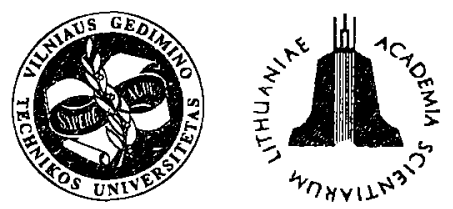

ISSN 1648-4142 TRANSPORT

http:/www.vtu.lt/english/editions

\title{
THE INVERSE SIMULATION STUDY OF AIRCRAFT FLIGHT PATH RECONSTRUCTION
}

\author{
Wojciech Blajer', Jacek A. Goszczyński ${ }^{2}$, Mariusz Krawczyk ${ }^{3}$ \\ ${ }^{1}$ Technical University of Radom, Poland, \\ 2, ${ }^{3}$ Institute of Aviation, Warszawa, Al. Krakowska 110/114 , 02-256 Warszawa, Poland
}

Received 200202 02; accepted 20020418

\begin{abstract}
This paper presents a uniform approach to the modelling and simulation of aircraft prescribed trajectory flight. The aircraft motion is specified by a trajectory in space, a condition on airframe attitude with respect to the trajectory, and a desired flight velocity variation. For an aircraft controlled by aileron, elevator and rudder deflections and thrust changes a tangent realization of trajectory constraints arises which yields two additional constraints on the airframe attitude with respect to the trajectory. Combining the program constraint conditions and aircraft dynamic equations the goveming equations of programmed motion are developed in the form of differential-algebraic equations. A method for solving the equations is proposed. The solution consists of time variations of the aircraft state variables and the demanded control that ensures the programmed motion realization.
\end{abstract}

Keywords: aircraft inverse dynamics and control, prescribed trajectory flight.

\section{Introduction}

Dynamic inversion (inverse simulation) is a systematic method of designing controllers for nonlinear control systems [1]. The problem involves first prescribing a desired motion and then determining the control inputs to a dynamic system that force the system to complete the prescribed motion. This can be especially useful for running unpiloted simulations, studying the required control strategies, and evaluating feasibility of the modelled aircraft maneuvers (or missions). In this paper inverse methods are applied to an aircraft trajectory prescribed path control problem. This is a topic of contemporary interest for the integrated design of guidance and control systems for autonomous/unmanned air vehicles. Most of the hitherto attempts, e.g. [2-5], used specified fuselage attitude slew maneuvers and flight velocity to define the performance goals. The four control inputs - deflections of aileron, elevator and rudder, and thrust changes - act then directly on the first derivatives of the four controlled variables and the control required to achieve these goals can readily be determined from an appropriate subset of the dynamic equations. In this way, however, the flight trajectory is neither directly specified nor controlled and the usual maneuver segmentation yields discontinuities in the motion specification at the transient points [4].

\footnotetext{
'e-mail:wblajer@poczta.onet.pl

2 e-mail: jagoszcz@ilot.edu.pl,

3.e-mail: krawczyk@ilot.edu.pl
}

The motion specification in this contribution directly includes a desired trajectory which is the most natural way to prescribe flight maneuvers. A specified flight path means two constraints on the position of aircraft mass center, and the two other constraints are a condition on the airframe attitude with respect to the trajectory and a specified flight velocity. An important feature of the trajectory constraints is that their realization is tangent $[6,7]$ - the available control inputs cannot directly regulate the balance of the applied and inertial forces in the constrained, i.e. orthogonal to trajectory directions. To achieve the balance without the actuating forces an appropriate adjusting of the fuselage attitude with respect to the trajectory is required. This means two additional constraints on airframe attitude (now regulated directly by the available controls) and can also be referred to as that the trajectory constraints are ,redoubled". The aircraft motion described in this way is thus fully specified and a, paradox" that an aircraft, a six-degree-of-freedom system, can explicitly be governed by four controls can also be explained.

\section{Mathematical Model of the Aircraft}

Choosing a hybrid coordinate system consisting of combined wind (or path) and body centroidal axes, the equations of motion of an aircraft take the following matrix form:

$$
m \dot{v}^{\left(w^{\prime}\right)}+m \widetilde{\omega}_{w^{\prime}}^{(w)} v^{\left(w^{\prime}\right)}=F^{(w)} ;
$$




$$
J \dot{\omega}^{(b)}+\tilde{\omega}^{(b)} J \omega^{(b)}=M^{(b)},
$$

where: $m$ and $J-$ vehicle mass and inertia matrix in the body axes; $v^{(w)}=\left[\begin{array}{lll}1 & 0 & 0\end{array}\right]^{T} v-$ vehicle inertial velocity representation in the wind axes; $\omega_{w}^{\left(w^{\prime}\right)}$ and $\omega^{(b)}=\left[\begin{array}{lll}P & Q & R\end{array}\right]^{T}$ - representations of the wind axes angular velocity $\vec{\omega}_{w}$ and the aircraft (body axes) angular velocity $\vec{\omega}$ respectively in the wind and body axes; and $\tilde{\omega}-$ a skew-symmetric matrix associated with $\omega$ to represent the vector product in matrix notation, e.g. the product $\vec{\omega}_{W} \times \vec{v}$ is represented in the path axes as $\tilde{\omega}_{w}^{(w)} v^{(w)}$. The applied forces and moments, $F^{\left(w^{\prime}\right)}$ and $M^{(b)}$, respectively in the wind and body axes, are:

$$
\begin{aligned}
& F^{(w)}=-\frac{1}{2} \rho S v^{2}\left[\begin{array}{c}
c_{D} \\
c_{S} \\
c_{L}
\end{array}\right]+T\left[\begin{array}{c}
\cos \left(\alpha+\alpha_{T}\right) \cos \beta \\
-\cos \left(\alpha+\alpha_{T}\right) \sin \beta \\
-\sin \left(\alpha+\alpha_{T}\right)
\end{array}\right]+ \\
& m g\left[\begin{array}{c}
-\sin \gamma \\
\sin \varphi \cos \gamma \\
\cos \varphi \cos \gamma
\end{array}\right] ;
\end{aligned}
$$

$M^{(h)}=\frac{1}{2} \rho S v^{2}\left[\begin{array}{c}b c_{l} \\ c_{a} c_{m} \\ b c_{n}\end{array}\right]+T\left[\begin{array}{l}0 \\ d \\ 0\end{array}\right]+J_{T} \omega_{T}\left[\begin{array}{c}-Q \sin \alpha_{T} \\ P \sin \alpha_{T}-R \cos \alpha_{T} \\ Q \cos \alpha_{T}\end{array}\right]$

where: $\rho$ - air density; $S, b$ and $c_{a}$ - lifting surface, wing span and mean aerodynamic chord; $\alpha$ and $\beta$ - angles of attack and sideslip; $\varphi, \gamma$ and $\chi-$ Bryant angles that describe the orientation of wind axes with respect to Earth axes; $T, \alpha_{T}$ and $d-$ thrust value, its inclination angle and line offset from the origin of wing/body axes; $J_{T}$ and $\omega_{T}=\omega_{T}(T)$ - moment of inertia and angular velocity of the jet rotating parts. The aerodynamic force and moment coefficients are the following functions of state variables and control surface deflections ( $\delta_{a}$ aileron, $\delta_{e}$ - elevator, $\delta_{r}$ - rudder):

$$
\begin{aligned}
c_{D} & =c_{D}\left(\alpha, \beta, \delta_{e}\right) ; & c_{l} & =c_{l}\left(\alpha, \beta, P, R, \delta_{u}, \delta_{r}\right) ; \\
c_{S} & =c_{S}\left(\alpha, \beta, P, R, \delta_{r}\right) ; & c_{m} & =c_{m}\left(\alpha, Q, \delta_{e}\right) ; \\
c_{L} & =c_{L}\left(\alpha, Q, \delta_{e}\right) ; & c_{n} & =c_{n}\left(\alpha, \beta, P, R, \delta_{u}, \delta_{r}\right) .
\end{aligned}
$$

\section{Motion Specifications}

The constraints used to generate the inverse control are specified as a desired trajectory in space, a demand on airframe attitude with respect to the trajectory and a desired velocity variation (Fig 1). These can be stated in the following forms:

$$
r=\bar{r}(s) \equiv\left[\begin{array}{c}
\bar{x}(s) \\
\bar{y}(s) \\
\bar{z}(s)
\end{array}\right]
$$

$$
\begin{aligned}
& \beta=\bar{\beta}(s) \text { or } \varphi=\bar{\varphi}(s) ; \\
& s=\bar{s}(t),
\end{aligned}
$$

where $s$ is the arc length parameter, and the superscript - means specified. The prescribed trajectory $\left(6_{\mathrm{a}}\right)$ is equivalent to two constraints on position of an aircraft treated as a particle in three-dimensional space. Two variant forms of the attitude constraint $\left(6_{\mathrm{b}}\right)$ are then allowed. The first one is most often taken as $\beta=0$, which characterizes a wide range of maneuvers that $v=\hat{v}(s)$ assume coordinated turns. The other form of $\left(6_{b}\right)$ can serve to model some aerobatic maneuvers like roll or bunt. Finally, the most natural constraint on flight velocity is $v=\widehat{v}(s)$. This can however always be transformed to the form $\left(6_{k}\right)$ by solving the following integral equation:

$$
\bar{v}(s) \Rightarrow \int_{s_{0}}^{\bar{s}(t)} \frac{d s}{\bar{v}(s)}=t \Rightarrow \bar{s}(t)
$$

In applications, the trajectory $\left(6_{a}\right)$ is first sketched by a set of successive points in space, and then interpolated/ approximated by spline functions. The foundations of such a procedure are described in [2]. The minimum requirement for the mathematical model that follows is that the functions $\bar{r}(s)$ must be at least twice differentiable. This suggests using spline functions of the third order [6] which may however lead to non-smooth variations of the obtained state and control variables [4]. So in the present work we used the fourth order splines, which considerably improved the obtained results (not reported here for shortness).

As it has already been mentioned, the realization of trajectory constraints $\left(6_{a}\right)$ is tangent $[6,7]$ - the available control inputs cannot directly regulate the balance of the applied and inertial forces in the orthogonal to trajectory directions. Instead, the balance must be achieved by appropriate adjusting the fuselage attitude with respect to the trajectory. This means two additional constraints on airframe attitude directly regulated now by coordinated

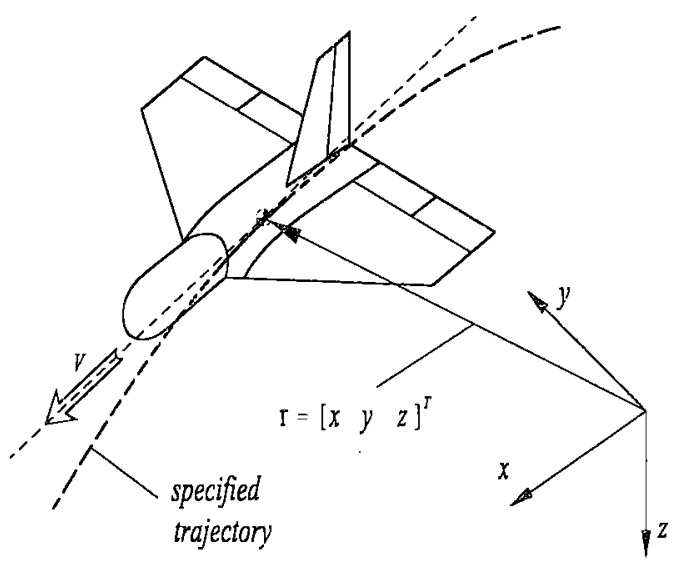

Fig 1. Prescribed trajectory flight 
control surface deflections. This can also be referred to as that the trajectory constraints are "redoubled" - the aircraft motion described by the four constraints (6) is thus fully specified. On the other hand, this also explains a "paradox" that at most four constraints can be imposed on an aircraft - a six-degree-of-freedom system, and that the system can explicitly be govern by the four controls $u=\left[\begin{array}{llll}\delta_{a} & \delta_{e} & \delta_{r} & T\end{array}\right]^{T}($ Fig 2).

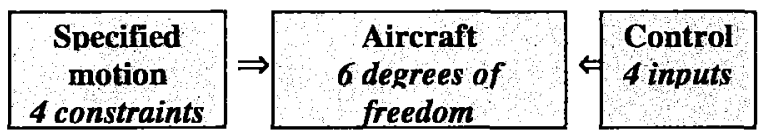

Fig 2. A,paradox"/consequence of tangent realization of trajectory constraints

\section{Governing equations}

The governing equations of aircraft prescribed trajectory flight can conveniently be written as the following set of twelve differential-algebraic equations (DAEs) [4]:

$0=F(y, z, u, t)-$ six modified conditions of constraints (6);

$z=G(y, \dot{y})-$ three kinematical differential equations; (9)

$z=H(y, z, u, t)-$ three dynamic equations (2),

where $y=\left[\begin{array}{lllll}\alpha & \beta & \varphi & \gamma & \chi\end{array}\right]^{T}, \quad z=\left[\begin{array}{lll}P & Q & R\end{array}\right]^{T}, \quad$ and $u=\left[\begin{array}{llll}\delta_{a} & \delta_{e} & \delta_{r} & T\end{array}\right]^{T}$ are respectively the position, velocity, and control variables. The explicit forms of equations (8) are:

$$
\begin{aligned}
& \beta=\widehat{\beta}(\bar{s}) \text { or } \varphi=\hat{\varphi}(\widehat{s}) \\
& {\left[\begin{array}{c}
\cos \gamma \cos \chi \\
\cos \gamma \sin \chi \\
-\sin \gamma
\end{array}\right]=\left[\begin{array}{c}
\bar{x}^{\prime} \\
\bar{y}^{\prime} \\
\bar{z}^{\prime}
\end{array}\right]}
\end{aligned}
$$

$0.5 \rho S v^{2} c_{D}-T \cos \left(\alpha+\alpha_{T}\right) \cos \beta+m g \sin \gamma_{A}+$

$+m \dot{v}=0$

$$
\begin{aligned}
& 0.5 \rho S v^{2} c_{S}+T \cos \left(\alpha+\alpha_{T}\right) \sin \beta-m g \sin \varphi \cos \gamma+ \\
& +m v^{2}\left[\bar{x}^{\prime \prime}(\sin \varphi \sin \gamma \cos \chi-\cos \varphi \sin \chi)+\right. \\
& +\bar{y}^{\prime \prime}(\sin \varphi \sin \gamma \sin \chi+\cos \varphi \cos \chi)+ \\
& \left.+\bar{z}^{\prime \prime} \sin \varphi \cos \gamma\right]=0 \\
& 0.5 \rho S v^{2} c_{L}+T \sin \left(\alpha+\alpha_{T}\right)-m g \cos \varphi \cos \gamma+ \\
& +m v^{2}\left[\bar{x}^{\prime \prime}(\cos \varphi \sin \gamma \cos \chi+\sin \varphi \sin \chi)+\right. \\
& +\bar{y}^{\prime \prime}(\cos \varphi \sin \gamma \sin \chi-\sin \varphi \cos \chi)+ \\
& \left.+\bar{z}^{\prime \prime} \cos \varphi \cos \gamma\right]=0,
\end{aligned}
$$

where the superscript ' means derivative with respect to $s$, and $\left(11_{b}\right)$ stands for two equations for the determination of $g$ and c. Equations (9) and (10) read then as follows [4]:

$$
\left[\begin{array}{c}
P \\
Q \\
R
\end{array}\right]=A_{b w}(\alpha, \beta)\left(B_{w}(\varphi, \gamma)\left[\begin{array}{c}
\dot{\varphi} \\
\dot{\gamma} \\
\dot{\chi}
\end{array}\right]+\left[\begin{array}{c}
\dot{\alpha} \sin \beta \\
\dot{\alpha} \cos \beta \\
-\dot{\beta}
\end{array}\right]\right)
$$

$$
\begin{aligned}
& {\left[\begin{array}{ccc}
J_{x} & 0 & -J_{x y} \\
0 & J_{y} & 0 \\
-J_{x y} & 0 & J_{z}
\end{array}\right]\left[\begin{array}{l}
\dot{P} \\
\dot{Q} \\
\dot{R}
\end{array}\right]+} \\
& {\left[\begin{array}{ccc}
0 & -R & Q \\
R & 0 & -P \\
-Q & P & 0
\end{array}\right]\left[\begin{array}{ccc}
J_{x} & 0 & -J_{x y} \\
0 & J_{y} & 0 \\
-J_{x y} & 0 & J_{z}
\end{array}\right]\left[\begin{array}{l}
P \\
Q \\
R
\end{array}\right]=M^{(b),}}
\end{aligned}
$$

where

$$
\begin{aligned}
& A_{b w}=\left[\begin{array}{ccc}
\cos \alpha \cos \beta & -\cos \alpha \sin \beta & -\sin \alpha \\
\sin \beta & \cos \beta & 0 \\
\sin \alpha \cos \beta & -\sin \alpha \sin \beta & \cos \alpha
\end{array}\right], \\
& B_{w}=\left[\begin{array}{ccc}
1 & 0 & -\sin \gamma \\
0 & \cos \varphi & \sin \varphi \cos \gamma \\
0 & -\sin \varphi & \cos \varphi \cos \gamma
\end{array}\right] .
\end{aligned}
$$

\section{Numerical Procedure}

The solution to DAEs $(8) \div(10)$, given explicitly in (11) $\div(13)$, are time-variations of state variables $y(t)$ and $z(t)$ in the prescribed motion, and of control $u(t)$ that assures the realization of the motion. Using the simplest Euler backward difference approximation method [8] the following procedure for solving the DAEs can be proposed. Given $y_{n}, z_{n}$ and $u_{n}$ at time $t_{n}$, the values $y_{n+1}$, $z_{n+1}$ and $u_{n+1}$ at time $t_{n+1}=t_{n}+\Delta t$ can be found as a solution to the following set of non-linear algebraic equations:

$$
\begin{aligned}
& 0=F\left(y_{n+1}, z_{n+1}, u_{n+1}, t_{n+1}\right) ; \\
& z_{n+1}=G\left(y_{n+1}, \frac{y_{n+1}-y_{n}}{\Delta t}\right) ; \\
& z_{n+1}-z_{n}=\Delta t H\left(y_{n+1}, z_{n+1}, u_{n+1}, t_{n+1}\right) .
\end{aligned}
$$

In this way the solution can be advanced from time $t_{n}$ to $t_{n+1}$. The Euler code can possibly be replaced by more accurate higher-order backward difference approximation methods [5].

\section{Case Study}

The basic application of discussed method for synthesis automatic jet aircrafts control system became veri- 
fied across realisation some numerical simulations, in which favourably one projected system in different conditions and at different nominal trajectories was verified.

The results of numerical simulation of modelled M96 Iryda jet aircraft loiter flight are shown in Fig $3 \div 7$. In the

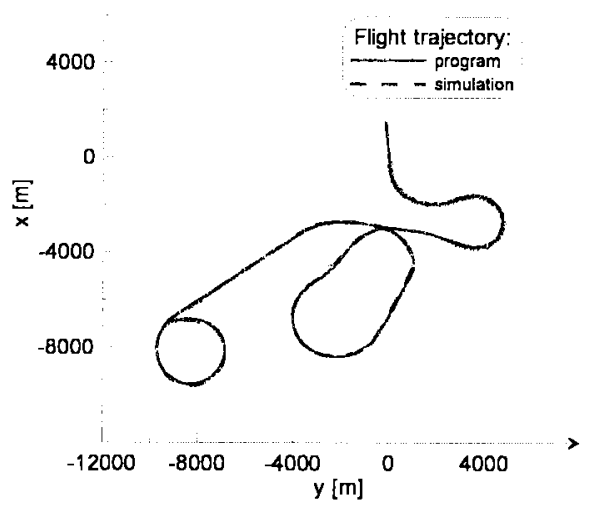

Fig 3. Trajectory
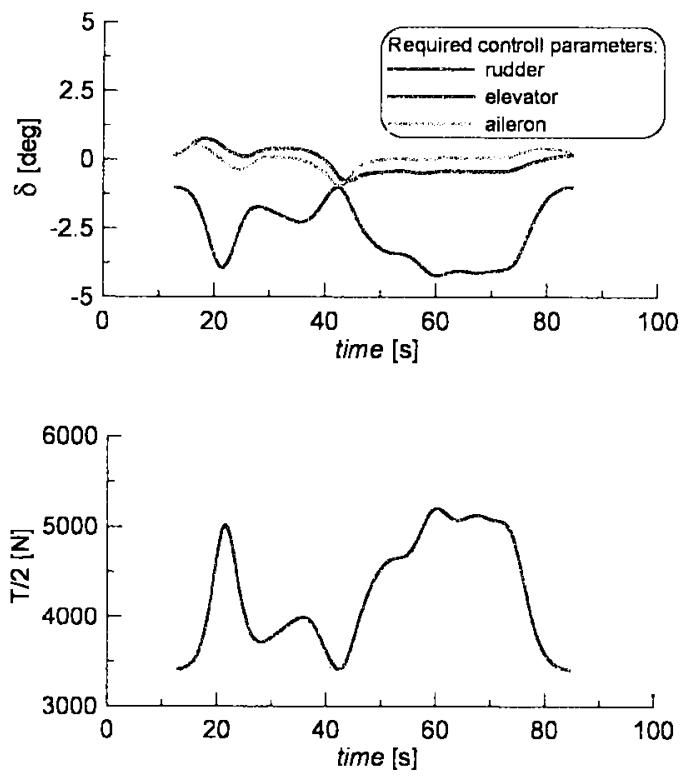

Fig 4. Control deflection

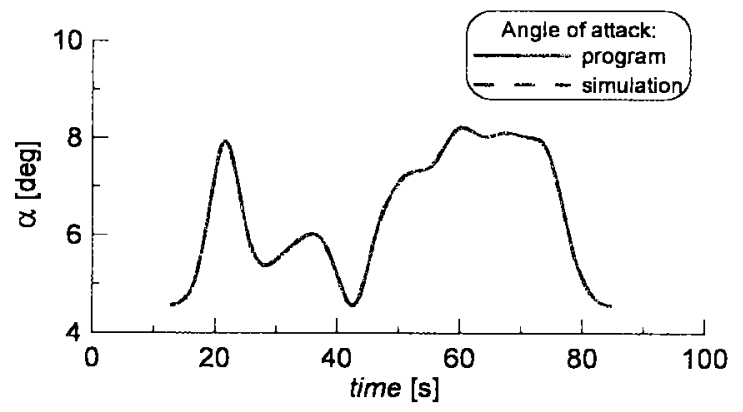

Fig 5. Angle of attack considered case, the trajectory, shown in Fig 3 was sketched by few points and then interpolated threw this points by $4^{\text {th }}$-order spline functions. The aircraft was then demanded to fly along the modelled trajectory at constant: velocity $V=130 \mathrm{~ms}^{-1}$ and altitude of flight $103 \mathrm{~m}$. The
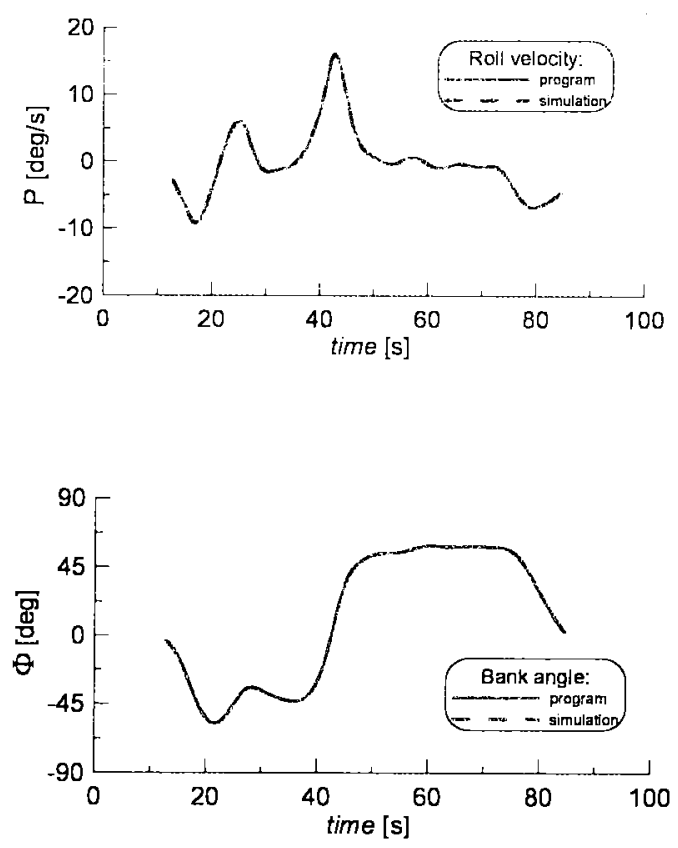

Fig 6. Angular velocity and appropriate roll angle
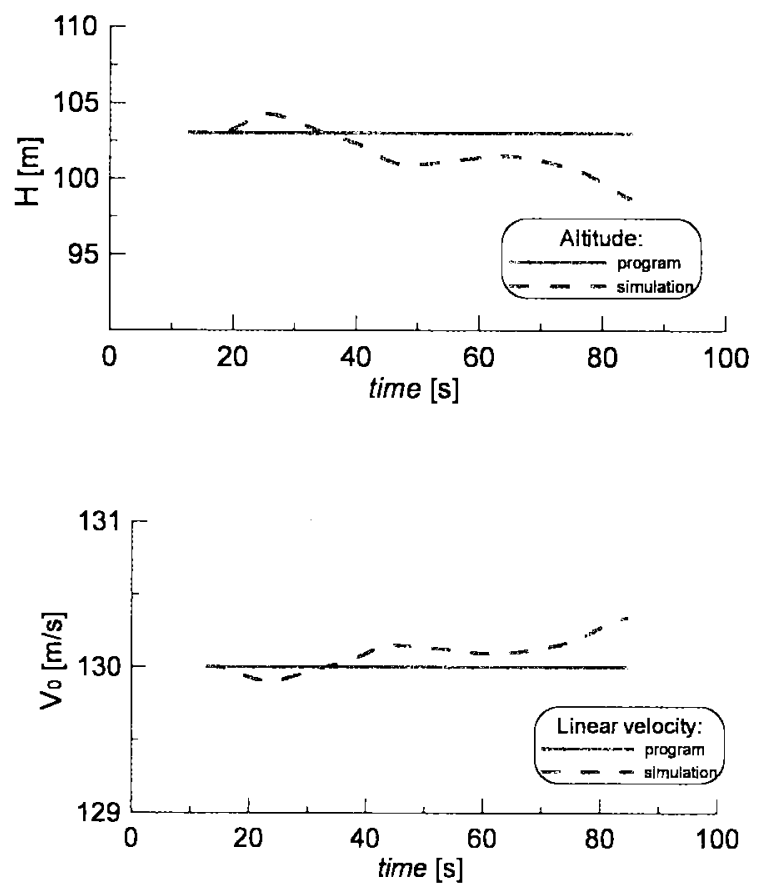

Fig 7. Velocity and altitude 
condition of coordinated turns $(\bar{\beta}=0)$ was assumed.

The obtained variations of nominal control $\delta_{a} \delta_{e} \quad \delta_{r} T$, shown in Fig 4, were calculated according (14).

The results for angle off attack $\alpha$ as well as the angular velocity $P$ and appropriate roll angle $\varphi$ are shown respectively in Figs 5 and 6.

The results for altitude $H$ and following linear velocity $V_{0}$ are shown in Fig 7 . The successful results of the research allow to build one's hopes that obtained inverse control variations can be used as powerful tool in the reconstruction of flight events/accidents.

\section{Final Remarks and Conclusions}

The achievements of this paper and the other experiences of the authors $[6,7]$, related to the subject in hand, can be summarized in the following six points:

1. A uniform mathematical model for aircraft prescribed trajectory flight simulation has been presented. The obtained solution consists of:

- time-variations of state variables in the prescribed motion,

- time-variations of the demanded control.

2. A simple numerical procedure for solving the followed governing differential-algebraic equations has been proposed and tested (not reported here).

3. There are at least three fields of direct application of the developed model:

- simulation and analysis of nature and feasibility (controllability) of a wide range of aircraft maneuvers, including extreme flight conditions and aerobatic maneuvers,

- design of unmanned aerial vehicles missions and their simulation,

- reconstruction of flight events/accidents.

4. The obtained inverse control variations can be used as control inputs (an open-loop tracking control) to aircraft in prescribed trajectory flight.

5. The inverse control should be enhanced by the addition of an appropriate feedback control. Only the resulting composite control will provide stable tracking of the required reference trajectories in the presence of perturbations and uncertainties in the aircraft dynamics. A linearized feedback can be developed as a minor extension of the proposed inverse control (not reported here for shortness).

6. The ongoing research on the project is focussed on three main problems:

- efficient numerical modelling of aircraft prescribed trajectories using 4th-order spline functions,

- design of linearized feedback control to enhance the inverse control,

- verification of the developed dynamic models and control laws.

\section{References}

1. Isidori A. Nonlinear control systems. Springer-Verlag, New York, 1989.

2. Azam M., Singh S. N. Invertibility and trajectory control for nonlinear maneuvers of aircraft. Journal of Guidance, Control, and Dynamics, 17, 1994, p 192-200.

3. Kato O., Sugiura I. An interpretation of aircraft general motion and control as inverse problem. Joumal of Guidance, 9 , 1986, p 198-204.

4. Lane S. H., Stengel R. F. Flight control design using nonlinear inverse dynamics. Automatica, 24, 1988, p 471-483.

5. Snell S. A., Stout P. W. Flight control law using nonlinear dynamic inversion combined with quantitative feedback theory. Journal of Dynamic Systems, Measurement, and Control, 120, 1998, p. 208-215.

6. Blajer W. A projection method - theory and applications in the analysis of constrained mechanical systems, Wyd. WSI w Radomiu, Radom, 1994.

7. Blajer W. Dynamics and control of mechanical systems in partly specified motion. Journal of the Franklin Institute, 334B, 1997, p 407-426.

8. Gear C. W., Petzold L. R. ODE methods for the solution of differential/algebraic equations. SIAM Journal on Numerical Analysis, 21, 1984, p 716-728. 